\title{
Photo- and Sono-dynamic therapy: A review of mechanisms and considerations for pharmacological agents used in therapy incorporating light and sound
}

\author{
Yanye Yang a , Juan Tu ${ }^{* a}$, Dongxin Yang ${ }^{a}$, Jason L. Raymond ${ }^{* b c}$, Ronald A. Roy ${ }^{\text {abc }}$ and Dong Zhang ${ }^{\text {a }}$ \\ ${ }^{a}$ Key Laboratory of Modern Acoustics (MOE), Department of Physics, Collaborative Innovation Center of \\ Advanced Microstructure, Nanjing University, Nanjing 210093, China; ${ }^{b}$ Department of Engineering Science, \\ University of Oxford, Oxford, United Kingdom; ' Oxford-Suzhou Centre for Advanced Research, Suzhou, \\ China
}

\begin{abstract}
As irreplaceable energy sources of minimally invasive treatment, light and sound have, separately, laid solid foundations in their clinic applications. Limited by relatively shallow penetration depth of light, traditional therapeutic modality of photodynamic therapy (PDT) usually involves superficial targets such as shallow seated skin conditions, head and neck cancers, eye disorders, early stage cancer of esophagus, etc. For ultrasound-driven sonodynamic therapy (SDT), however, accessibility to various organs is permitted by superior transmission and focusing ability of ultrasound in biological tissues, enabling multiple therapeutic applications including treating glioma, breast cancer, hematologic tumor and opening blood-brain-barrier (BBB), etc. Considering the newly-emerged theranostics and precision therapy, these two classic energy sources and corresponding sensitizers are worth re-evaluating. In this review, three typical therapies using light and sound as trigger, PDT, SDT, and combined PDT and SDT are introduced. The therapeutic dynamics and current designs of pharmacological sensitizers involved in these therapies are presented. By introducing both the history of the field and the most up-to-date design strategies, this review provides a systemic summary on the development of PDT and SDT, and fosters inspiration for researchers working on 'multi-model' therapies involving light and sound.
\end{abstract}

Keywords: Photodynamic therapy, Sonodynamic therapy, Sono-photodynamic therapy, Perfluorocarbon, Photosensitizer, Sonosensitier, Ultrasound Contrast Agents

\section{INTRODUCTION}

Light and sound have both been utilized as sources of energy for minimally-invasive treatment in clinics, namely high intensity focused ultrasound (HIFU) ablation of tumors (malignant and benign)[1], interstitial laser thermotherapy[2] and multiple eye surgeries for correction of refractive error. With the rapid development of light source control and photosensitizer (PS) advancements[3], clinical applications of PDT have expanded to include both malignant diseases[4] and non-malignant skin conditions[5, 6]. However, the shallow penetration depth of light (the optical penetration depth into skin is approximately 1.5, 1.8, 2.2 and $2.4 \mathrm{~mm}$ for wavelengths of 600,660, 750 and $850 \mathrm{~nm}$ )[7] imposes a severe limitation on traditional PDT in terms of delivering sufficient energy to deep seated targets. To overcome such barrier, researchers on the one hand explored the possibility of PS modification to achieve better therapeutic outcome

*Address correspondence to this author at the Key Laboratory of Modern Acoustics (MOE), Department of Physics, Collaborative Innovation Center of Advanced Microstructure, Nanjing University, P.O. Box:210093, Nanjing, China; Tel/Fax: +86-25-83594184, +86-25-83315557; E-mail: juantu@nju.edu.cn

*Address correspondence to this author at the Department of Engineering Science, University of Oxford, 17 Parks Road Oxford OX1 3PJ, United Kingdom; Tel/Fax: +44-1865-283265, +44-1865-273010; E-mail: jason.raymond@eng.ox.ac.uk
[8, 9]. On the other hand, while ultrasound has the ability to transmit to a much greater depth in biological tissues and can be controlled and spatially localized, it has also been found to be able to excite the same types of PS and generate similar therapeutic effects as PDT[10,11]. For those sensitizers that respond both to ultrasound and laser stimulation, the effect of sono-photodynamic therapy (SPDT) was proven even better than single-source irradiation[12, 13]. Alongside PDTderivated chemical-based SDT, ultrasound itself can also induce cell death in localized areas by mechanical stress and thermal effects with the help of ultrasound-sensitive substances, many of which could be modified with targeting molecules. Such therapeutic characteristics of ultrasound can enable synergic effects, providing the possibility of combined therapy[14, 15].

Up to date reviews concerning PDT and SDT mostly focus on single source irradiation, that is, to use only light or ultrasound to trigger therapy. The principles of sensitizer development and clinical translation advances of both PDT [4-6,16-19] and SDT[20-23] have been elaborately reviewed by several researchers. However, despite that PDT and SDT are often considered separate branches of therapies, it is suggested that combined SPDT may have even more clinical translation potential with its lowered incident energy levels[24]. Moreover, although researchers on both sides have devoted great effort in developing high-efficiency 

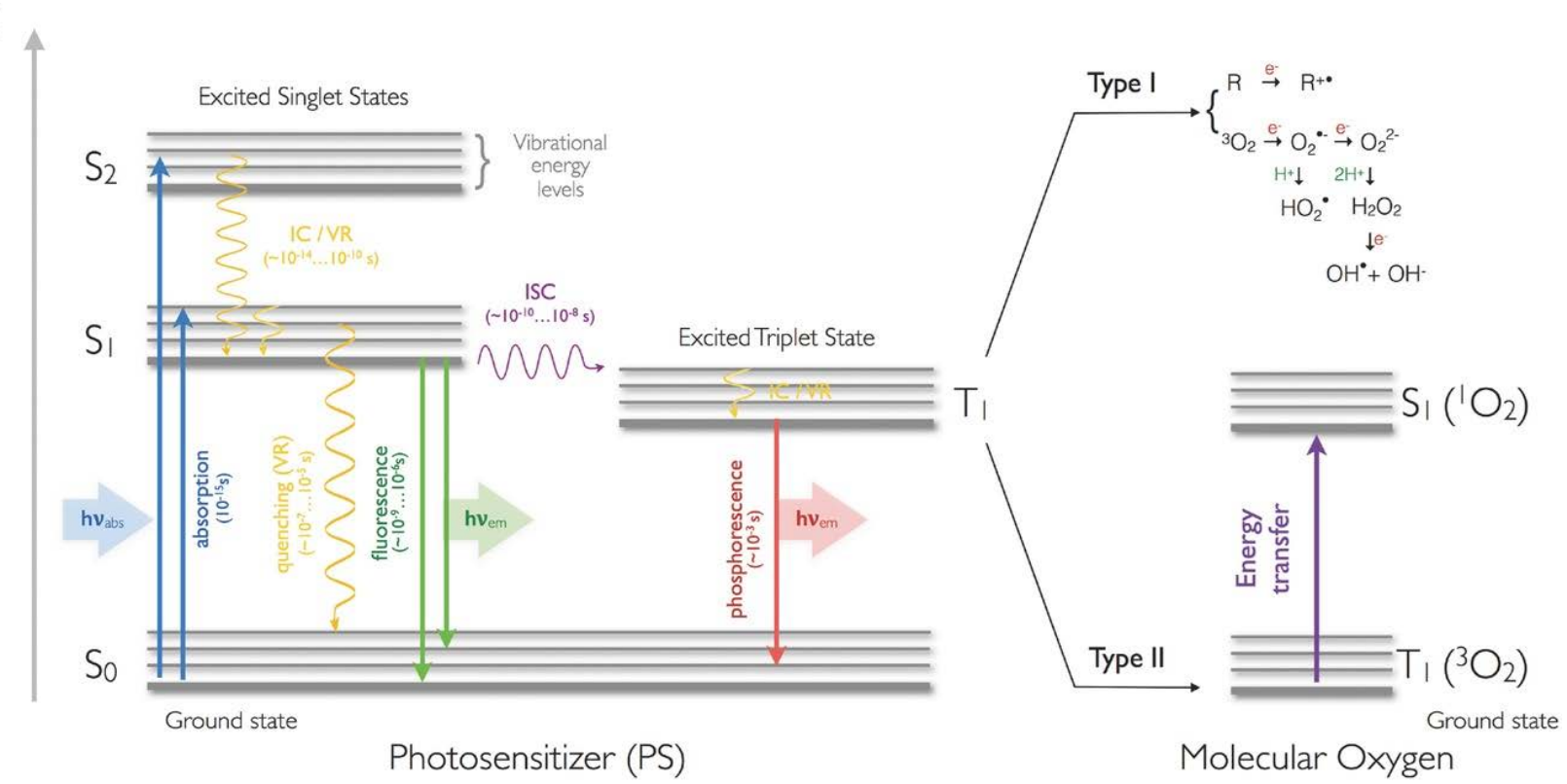

Fig. 1. Photochemistry and photophysics illustrated by Jablonski diagram. From Lismont et al [3]. Copyright @2017 Advanced Functional Materials, reproduced with permission.

sensitizers separately, it is clearly demonstrated in this review that PDT and SDT share many aspects of development and application in common. It is thus beneficial to set a common ground where researchers on both sides may find inspiration in each other's achievements. In this article, the mechanisms for and development of PDT, chemical agent based sonodynamic therapy, and nonchemical based sonodynamic therapy are reviewed. Strategies for the enhancement of PDT and SDT outcomes are introduced, focusing mainly on advances in sensitizers. By introducing latest developments of pharmacological agents used in therapy incorporating light and sound, this review aims to hatch new thoughts for sensitizer design and combined therapy.

\section{PHOTODYNAMIC THERAPY (PDT)}

\subsection{The principle of photodynamic therapy (PDT):}

PDT refers to induced cellular toxicity proceeding from the photodynamic effect of light-activated dyes. As a minimally invasive treatment, PDT has been widely utilized in treatments of malignant diseases[4], eye disorders (agerelated macular degeneration[25], keratoconus [26]), wound healing, and other shallow-seated skin conditions (chronic ulcers, non-melanoma skin cancer, acne, cutaneous leishmaniasis etc.)[5, 6]. In PDT, a light-activated dye (usually referred to as a 'PS' is irradiated with a monochromatic light source that matches the absorption spectrum of the dye. The irradiated ground state PS loses energy spontaneously through fluorescent emission or heat.
In some cases, excited singlet state PS may transit to excited triplet state after passing through intersystem crossing (ISC) [3]. Upon relaxation from this state, the excited triplet state PS can trigger two types of photochemical reactions, as illustrated in Figure 1. In Type 1, it reacts directly with organic molecules in its proximity or molecular oxygen, thus forming radical anion/cations by electron/proton transfer and the initial generation of superoxide anion. In Type 2, energy is transferred to ground-state triplet molecular oxygen directly from the excited triplet-state PS, resulting in the production of excited-state singlet oxygen. The two types of reactions can take place simultaneously, with their ratio dependent on the type of PS as well as the amount of surrounding organics and oxygen[18].

PDT affects cells by altering or deactivating signal transduction pathways, by oxidizing biological molecules, and by arousing cellular stress responses (hypoxia and angiogenesis). Those effects may induce cell apoptosis, necrosis and cellular DNA damage (DNA-based oxidative damage, strand breaks and cross-links), the mechanisms and prerequisites of which have been widely reported[19, 27-29]. In addition to the cytotoxic effects of PDT-produced reactive oxygen species (ROS) on cells, PDT exhibits two other mechanisms that contribute to tumor reduction/destruction: (i) PDT-induced thrombosis blocks oxygen and nutrient supply and induces hemorrhage in tumor vasculature and (ii) it causes leukocytes, lymphocytes and macrophages to penetrate into the treated area, thereby stimulating immune response against the tumor[4, 27]. 


\subsection{Photosensitizers (PSs):}

Commonly used PSs can be roughly categorized into porphyrins, phthalocyanines (Pcs) and BODIPY dyes[30]. Biosynthetic porphyrins were first to be developed, resulting in the commercial product Photofrin, a hematoporphoyrin derivative approved for cancer treatment in Canada and United States in the 1990s, thus marking the birth of first generation PS. Pcs, the second generation of PS, absorb light at a longer wavelength (with the longest absorption band in $>650 \mathrm{~nm}$ and enhanced molecular extinction coefficient of around $10^{5} \mathrm{~L} \mathrm{~mol}^{-1} \mathrm{~cm}^{-1}$ ) than most porphyrins (with the longest absorption band in around $630 \mathrm{~nm}$ and molecular extinction coefficient of around $5 \times 10^{3} \mathrm{~L} \mathrm{~mol}^{-1} \mathrm{~cm}^{-1}$ ) , resulting in deeper optical penetration into tissues and increased singlet oxygen production while relieving the skin photosensitivity problems[18, 30, 31]. BODIPY dyes, the third generation of PS, exhibit increased solubility by combining PS with nanoparticles, which enables PS to cross biological barriers[3]. More recently, researchers have been focusing on making multi-responsive and multi-functional PS, which might be considered as the fourth generation of PSs. For example, based on the slightly acidic microenvironment of tumor, Ju et al. developed a $\mathrm{pH}$ sensitive theranostic PDT agent that can selectively generate singlet oxygen and emit near infrared (NIR) fluorescence under slightly acidic conditions[32]. Battogtokh et al. combined folate with PS PheA and acid-responsive linker cisaconityl, aiming at acid responsive PS release and higher tumor targeting efficacy[33]. Mironova et al. synthesized genetically encoded PS, which consisted of high-efficient recombinant $4 \mathrm{D} 5 \mathrm{scFv}$ mini-antibody and phototoxic protein miniSOG (mini Singlet Oxygen Generator) so as to enable selective cytotoxicity to HER2/neu-overexpressing cells[8]. To address the limitation of shallow penetration depth of light, a new type of PS, namely upconversion nanoparticles (UCNPs), was extensively explored, increasing the depth of optical diagnosis and therapy with advantageous nonradiative excitation energy transfer capability[9].

\subsection{Hypoxia in photodynamic therapy (PDT) and Oxygenation Solutions:}

\subsubsection{Perfluorocarbon (PFC) as the oxygen container:}

The local oxygen level is an important determinant of PDT efficacy for treatments based on ROS generation. Due to reduced oxygen supply in tumors, which can be further aggravated by PDT, however, the therapeutic effect of PSs is often compromised[34-36]. To overcome this limitation, attempts have been made to maintain/increase the local oxygen levels by adjusting fluence rate or fractionating light irradiation[37, 38], by inducing hyperthermia[39-41], by hyperbaric oxygenation[42, 43], and by the application of oxygen-generating particles (e.g. PFCs)[44, 45].

The high oxygen solubility of PFCs made them promising candidates as artificial blood substitutes, which can be traced back to 1966 when Clark and Gollan reported successful respiration support provided by PFCs in mice[46]. Since then, extensive trials and products of various formulae have demonstrated PFCs' potential as an oxygen-delivering agent, which has been elaborately reviewed by Castro et al.[47]. Injecting PFC emulsions to the treatment area may increase local oxygen availability and increase the efficacy of PDT treatment, especially in hypoxic environment. As early as 1988, Fingar et al. tried to co-inject carbogenequilibrated Fluosol-DA (a clinically used blood replacement perfluorochemical emulsion, which was later withdrawn by the FDA in 1994 due to storage and pre-usage difficulties[48]) and a PS called Photofrin II for PDT[49]. Recently, other methods of co-administration of PFCs and PSs have been proposed and many of them have shown elevated PDT efficacy[44, 45, 50, 51]. Figure 2 shows a typical basic structure of an oxygen container for enhanced PDT.

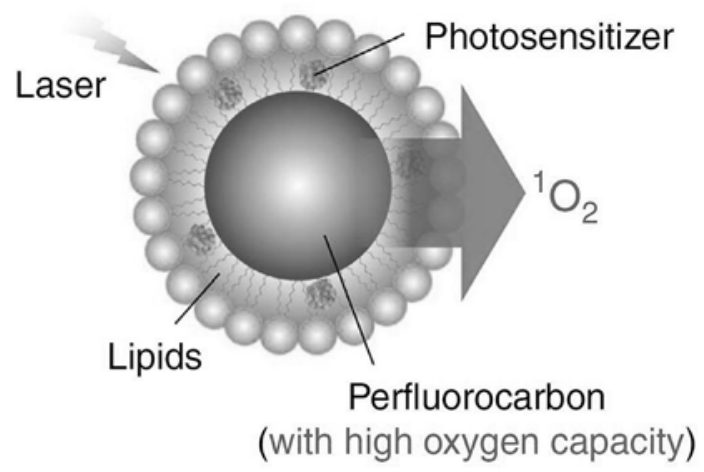

Fig. 2. Typical design of oxygen self-enriched agent for PDT. From Cheng et al [45]. Copyright @2015 Nature Communications, reproduced with permission.

In 2013, Wang et al. hypothesized that the singlet oxygen generation capacity of an Indocyanine green (PS)-loaded perfluoro-15-crown-5-ether (PFC) nanoemulsion could be significantly higher than that of free Indocyanine green under laser irradiation, due to the increased solubility of oxygen in PFC[50]. Sheng et al. introduced a therapeutic and imaging agent based on Indocyanine green and perfluorooctyl bromide (PFOB), an oxygen-enriching PFC[52]. It was proposed that PFOB not only played a crucial role in elevating PDT efficacy by increasing singlet oxygen generation, but also helped to further enable photothermal therapy (PTT) by reducing the expression of heat shock protein (HSP). In 2015, Cheng et al. incorporated perfluorohexane (PFH) and PS IR780 into a lipid-shell to form an oxygen self-enriched nanodroplet for PDT, namely LIP (IR780 + PFH)[45]. The treatment efficacy of LIP (IR780 +PFH) was shown to be superior than the PFH-free agent in both cell-assays and in vivo studies. With intratumoral injection, tumor volumes were significantly reduced for mice treated with LIP (IR780 +PFH) plus laser irradiation whereas tumor volumes were not significantly reduced during two-weeks' period post-treatment in other groups (saline control group, LIP (IR780 +PFH) alone, and LIP (IR780) with/without laser irradiation included). With intravenous injection, a fourfold inhibition effect could be established by LIP (IR780 +PFH) plus laser irradiation in comparison with the PFH-free agent plus laser irradiation by day 10 post-treatment. 
More recently, Wang et al. synthesized a versatile theranostic Oxy-PDT agent for fluorescence/photoacoustic (PA)/X-ray computed tomography imaging and laserinduced PTT combined with oxygen-enriched PDT[53]. By measuring the size of tumor, it was shown that combined PTT \& PDT achieved complete tumor inhibition 40 days post-injection. In addition to the therapeutic efficacy of the synthesized agent, its PA diagnostic imaging ability was also of great importance. By acquiring time-dependent PA images, the location of the tumor and the accumulation rate of this agent can be visualized in real-time, which suggests the possibility of PA image-guided therapy.

In 2016, Song et al. demonstrated that $1 \mathrm{MHz}$ ultrasound can trigger burst oxygen release from human serum albumin (HSA) encapsulated, oxygen-enriched PFC nano-emulsion both in vitro and in vivo[54]. By triggering oxygenation within the tumor using a PFC nano-emulsion agent and ultrasound exposure for 30 minutes before the application of traditional PDT/radiotherapy (RT), significant reduction was found in average tumor weight not only in comparison with untreated mice, but also with mice treated solely with $\mathrm{PDT} / \mathrm{RT}$.

\section{CHEMICAL AGENT BASED SONODYNAMIC THERAPY (SDT)}

\subsection{The principle of chemical agent based sonodynamic therapy (SDT):}

SDT, in a narrow sense, involves ultrasound activated cytotoxic ROS generation. Similar to PDT, the basic elements required for SDT includes a chemical (sonosensitizer or ultrasound-active chemotherapy drug), oxygen and ultrasound[55]. The activation process is generally considered to be induced by cavitation, which could further induce sonoluminescence, cell apoptosis, and pyrolysis, while the dominant effect still needs to be analyzed based on the formulation and exposure conditions[56-58]. Two major sonochemical products arise from ultrasonically-induced cavitation: free radicals and singlet oxygen. Free radicals can induce chain reaction of lipid peroxidation as well as cell damage while singlet oxygen, once entering excited singlet state, is capable of oxidizing cellular contents.

Because it is also dependent on the local availability of oxygen, SDT also faces the similar obstacle as PDT, that is oxygen deficiency in treated area. To overcome this, a similar self-oxygenation approach was proposed. In 2017, Chen et al. developed a mesoporous organosilica PFC-chainchelated nano-vehicle for ultrasound-responsive oxygen delivery and SDT[59]. In this design, the organosilica shell was modified with PFC chains for oxygen capture, and the sonosensitizer was loaded through electrostatic interaction. Upon ultrasound irradiation, this agent exhibited continuous oxygen release, which could largely relieve the hypoxia milieu. For nude mice bearing PANC-1 pancreatic tumor, decrease in tumor volume and prolonged survival time were observed after three repeated SDT treatments. Interestingly, Chen et al. found that the oxygen-enriched agent alone caused more cell death than the non-oxygenated agent combined with ultrasound irradiation in vitro, which reemphasized the importance of sufficient oxygen in SDT[59]. The authors ascribed this effect to simultaneous oxygen release by the oxygen-enriched agent, which could further enable continuous ROS production 12 hours post-treatment due to sustained hyperbaric oxygen beneficial.

SDT has become a new solution in cancer therapy not only due to the direct effect of tumor inhibition, but also due to the synergic effects with chemotherapy and other forms of regular treatment[58,60].

\subsection{Sonosensitizers:}

\subsubsection{Porphyrins and their derivatives:}

As the first FDA approved therapeutic agent for PDT, porphyrins were also among the first sonosensitizers investigated for SDT applications. In 1989, Umemura et al. proposed that hematoporphyrin (Hp) can increase the sensitivity of tumor cells to ultrasound irradiation, the mechanism being ultrasound-triggered singlet oxygen generation[10, 61]. The inhibition effect was further confirmed in vivo in sarcoma 180 bearing mice exposed to $1.92 \mathrm{MHz}$ ultrasound at the intensity of $1.70 \mathrm{~W} / \mathrm{cm}[11]$. Tumor growth suppression of $73.6 \%$ was found for mice treated with both ultrasound and $\mathrm{Hp}$, while $\mathrm{Hp}$ and ultrasound alone showed minimal inhibition effect (2.4\% and $14.9 \%$ respectively). Since then, numerous experiments have been run employing different cell lines, ultrasound parameters, and sonosensitizers based on porphyrin derivatives. Alongside the cytotoxic effects and tumor size reduction effects, other aspects of SDT were also explored to further understand this process. In 1994, Cain et al. reported that no correlation can be found between the efficacy of a certain porphyrin under light and ultrasound irradiation[62]. Wei Tang et al. assessed the changes of mitochondrial properties as well as apoptosis-related protein translocation, revealing the activation of mitochondrial pathway[63].

Hematoporphyrin monomethylethe (HMME), as a porphyrin derivative, has also been considered as a sonosensitizer for its tumor accumulation properties. In 2008, Jian-hua $\mathrm{Li}$ et al. successfully demonstrated the synergic effect of ultrasound and HMME, finding that HMMEmediated SDT can not only inhibit tumor growth, but also affect the cell cycle[64]. Five years later, Xiaomin Su et al. reported that the mitochondria-caspase-signaling pathway was activated during HMME-mediated SDT, which further induced cell apoptosis of U937 cells[65]. When combining cell necrosis induced by ultrasound cavitation with HMME mediated cell apoptosis, the achieved synergic therapeutic effects yielded a much higher tumor inhibition rate[66]. Yan et al. designed a HMME/ Poly (DLlactic-co-glycolic acid) (PLGA) microcapsule with PFC gas core. The structure/composition of such design was believed to play a crucial role in restraining cell proliferation rate through either superior HMME dispersion or cavitation effect brought by the gas core[67].

Another PS, protoporphyrin IX disodium salt (PpIX) was proven reactive to ultrasound, inducing apoptosis on HL-60 cells[65, 68]. In 2017, Ping Huang et al. built a multifunctional theranostic agent that encapsulated $\mathrm{Mn}$ chelated-PPIX, which is capable of both SDT and magnetic resonance imaging (MRI) enhancement. Both in vitro cell assessment and in vivo tumor treatment exhibited strong 
therapeutic effect with SDT induced by the synthesized agent, suggesting the possibility of clinical application[69].

Other porphyrin derivatives that have been proven effective in SDT include, but are not limited to pheophorbide A[70], photofrin[71], photofrin II[72, 73], ATX-70[74-76], ATX-S10[77, 78], Ce6[79] and DCPH-P-Na(I)[80, 81].

\subsubsection{Xanthene:}

Sonosensitizers that fit in this category mainly includes erythrosine B, rose bengal and their derivatives. Erythrosine $\mathrm{B}$ and rose bengal were proven effective in inducing sarcoma 180 cell damage in a $1.93 \mathrm{MHz}$ standing-wave ultrasound field, the mechanism of which could be the combined outcomes of sonochemical reactions and reduced cavitation threshold by erythrosine B/ rose-bengal[82, 83]. In a C6 glioma-bearing rat model, a massive reduction of tumor area was achieved by rose bengal injection and focused ultrasound irradiation[84]. To further improve the tumorspecific accumulation abilities, attempts were made to modify rose bengal in order to achieve higher therapeutic efficacy, many of which showed promising cytotoxic/tumor inhibition outcome[85, 86].

\subsubsection{Other sonosensitizers}

Other sonosensitizers mainly include other chemicals, inorganic nanoparticles, and complex nano-delivery platforms. Successful cytotoxicity and/or tumor inhibition has been established with ZnPcS2P2[87], porous silicon nanoparticles[88], fullerene[89] and emodin[90, 91]. A compound derived from a traditional Chinese herb, emodin was found effective in reinforcing ultrasound-induced apoptosis and was thus considered to be sonosensitizer[91]. The underlining mechanism of emodin-induced SDT was reported to be microtubule oxidation and subsequent cleavage[90].

In comparison with organic sonosensitizers, inorganic sonosensitizers are more chemically/physiologically stable, providing the possibility of modification, encapsulation and mass production[92]. $\mathrm{TiO}_{2}$, a recognized PS, was reported to be a sonosensitive agent that can generate ROS by itself upon ultrasound irradiation[92]. With a $10 \mu \mathrm{TiO}_{2}(0.500 \%)$ intratumor injection followed by ultrasound exposure of 2 minutes (1 $\left.\mathrm{MHz}, 1.0 \mathrm{~W} / \mathrm{cm}^{2}\right)$, tumor growth was significantly inhibited compared with either untreated mice or mice treated with only ultrasound or $\mathrm{TiO}_{2}$ [93]. Yamaguchi et al. compared the efficacy and cell-killing mechanism between PDT and SDT induced by PEG-modified $\mathrm{TiO}_{2}$ [94]. By treating U251 cells separately with SDT and PDT, it was found that SDT cytotoxicity was nearly proportional with ultrasound application time while PDT cytotoxicity only appeared above certain duration of UV irradiation. With the help of singlet oxygen scavenger glutathione and cell membrane fluorescent imaging, the authors concluded that, unlike PDT-induced cell death, SDT exhibits cytotoxic characteristics with the help of physical force exerted on the cell membrane by ultrasound rather than oxidative radicals produced by PEG-modified $\mathrm{TiO}_{2}$.

As $\mathrm{TiO}_{2}$ can appear not only in the form of solid particles but also as a mesoporous shell or a core of a complex composition, many researches have focused on integrating $\mathrm{TiO}_{2}$ into a multi-functional agent. Shen et al. coated $\mathrm{Fe}_{3} \mathrm{O}_{4}$ magnetic nanoparticles with $\mathrm{TiO}_{2}$ for chemotherapeutic drug doxorubicin (DOX) loading through electrostatic interaction[95]. The synergic effect of chemotherapy and SDT was substantial in tumor inhibition experiments, especially with the help of ex vivo magnet-assisted accumulation[95]. To realize image-guided, targeted sonodynamic-chemo synergic therapy, $\mathrm{NaYF}_{4}$ was added to the core composition of the aforementioned agent to enable up-conversion luminescence (UCL) imaging. The synthesized agent showed nucleus affinity in KB and MCF-7 cells, which might serve to promote DOX's intercalation and enhance cytotoxicity. The average tumor volume of mice treated with the agent after 24 hours of magnetic targeting decreased steadily after 4 treatment cycles, while other groups showed no inhibition effect[96].

\section{NON-CHEMICAL BASED SONODYNAMIC THERAPY(SDT)}

Despite the fact that the term 'SDT' was first proposed to describe the ultrasound activation of photochemical sensitizers[10, 11, 61], it can also be considered to include other forms of non-thermal ultrasound therapy as well[56]. As one of the most important biological effects of nonthermal ultrasound therapy, sonoporation and assisted gene/drug delivery are introduced in the following section.

\subsection{Sonoporation:}

The term sonoporation is used to describe the process whereby temporary pores are acoustically induced, through which the internalization of cell impermeable molecules into living cells could be realized[97]. The permeability can be enhanced with the presence of ultrasound contrast agents (UCAs), which typically consist of stabilized microbubbles[98]. The existing bubbles serve as cavitation nuclei, which will act under the incident acoustic pressure wave and transiently porate the cell membrane to make entrance for the released drug/gene molecules[99]. While ultrasound induced pore formation can be observed at different acoustic intensities, the mechanisms and prerequisites vary accordingly.

Depending on the driving acoustic pressure amplitude, microbubbles may oscillate linearly about an equilibrium radius, oscillate nonlinearly about an equilibrium radius (stable cavitation), or grow unstably and collapse violently (inertial cavitation). In some cases the pulsations may be non-symmetric depending on environmental factors such as asymmetries in the sound field or the proximity of other bubbles and/or boundaries[100].

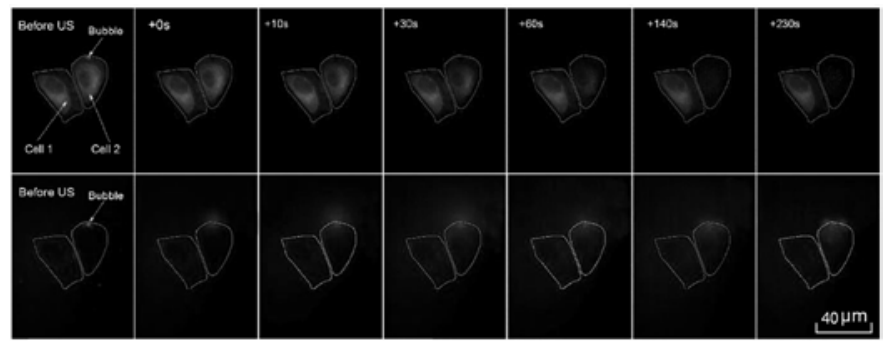

Fig.3. Cell membrane and cytoskeleton response under ultrasound irradiation. From Fan et al [104]. Copyright @2017 Theranostics, reproduced with permission. 
For stably cavitating microbubbles, sonoporation is thought to be induced by the shear stresses (imposed by bubble oscillation) as well as cavitation microstreaming (induced by said stresses in surrounding liquid)[97]. The extent to which these stresses impact a cell depends on the amplitude of incident ultrasound wave[101, 102] and the distance between cavitating bubble and cell[103]. Figure 3 shows real-time cellular response of HeLa cells induced by microbubble-mediated sonoporation. After ultrasound irradiation, the HeLa cell on the right exhibited $\alpha$-tubulin cytoskeleton disassembly and increased membrane permeabilization, which is indicated by diminishing green fluorescence and diffusing red fluorescence respectively[104]. de Jong and his colleagues studied the deformation of a cell membrane during sonoporation using high speed microscopy and reported that only in close vicinity to cells can bubbles perturb the cell membrane in a manner sufficient to yield increased permeability of propidium iodide (PI). Successful sonoporation was characterized by a pore that formed in the intact lipid structure of cell membrane and resealed when either the bubble was repelled or the ultrasound turned off[103]. This perforating and resealing process was visualized under confocal microscope by $\mathrm{Yu}$ et al., and the influence of temporal-peak pore size and extra-cellular calcium on pore resealing and its duration was explicitly explored[105].

For bubbles undergoing inertial cavitation, sonoporation is thought to be induced by bubble collapse and collapse microjets and shockwaves[97, 106-108]. Bubbles located close to a boundary (or each other) manifest asymmetric characteristics. For the violent collapses associated with inertial cavitation, an inward flow asymmetry forms, resulting a high-velocity liquid jet that traverses the bubble and impacts the boundary. In the case of inertial collapses that are sufficiently remote from boundaries, the rapid acceleration associated with the final instances of collapse serve to launch an outgoing spherical shock wave. Both effects are directly responsible for the penetration of a cell membrane and even cell detachment from its substrate[108110]. The process of laser-trapped microbubble cavitation at different distances from cell monolayer was visualized by Prentice et al in 2005, and results show pronounced disruption on cell membranes produced by microjetting[110]. In 2017, Fan et al reported that the degree of membrane disruption is cell-cycle-dependent due to different mechanical properties in different cycle phases[104].

Owing to the membrane-opening effect of sonoporation and the feasibility of gene/drug loading on microbubbles, contrast agents exhibit great potential to become a nextgeneration delivery vehicle[14]. The fact that microbubbles can survive blood circulation addresses the quick clearance problem of non-viral DNA vectors[111, 112]. Under ideal circumstances, a gene/drug that has been successfully attached or incorporated on the shell will be protected by the bubble shell structure during circulation, and once the bubble enters a malignant site, the loaded gene/drug could be locally released by ultrasound and enter the tumor cells via sonoporation. By using such methods of controlled release, both DNA payload and on-site delivery efficacy can be increased, resulting in significantly increased transfection efficacy[14, 113, 114].
DNA incorporation into bubbles could be realized through two approaches: physical linkage or electrostatic adsorption[115]. Physical linkage was first attempted by Teupe et al. and was confirmed to be effective by a vascular transfection study[116], a cell transfection study[113] and an in vivo study[117]. Electrostatic adsorption could be realized by cationic-anion reactions, which was proven viable by a cell transfection study[118], an in vivo tumor transfection assay[118], and by an in vivo muscle transfection study[119].

Due to the ultrasound-triggered local release properties of drug-loaded microbubbles, attempts have been made to load toxic chemotherapy drugs onto microbubbles, by which systematic toxicity and other side effects could be reduced. DOX, a potent chemotherapy drug, was widely used as a target bubble-loading drug due to its severe side effects[120]. By simply mixing DOX with a rehydrated lipid solution (together with EDTA and glucose) during bubble formation, an $87.3 \%$ loading rate could be achieved[121]. Further studies showed a 12-fold increase in DOX concentration in tumor areas than in the contralateral side, indicating increase local drug delivery efficacy[120]. In 2013, Wu et al. chemically conjugated DOX with Pluronic F68 and synthesized targeted DOX-loaded microbubbles with bFGF. Through tumor volume measurement, targeted DOX-loaded microbubbles significantly inhibited tumor growth with minor side effects in comparison with either free DOX or free DOX-Pluronic[15]. Despite the widely accepted concept that the drug molecules enter cell membrane through pores created by sonoporation, De Cock et al. proposed that rather than passive diffusion and endocytosis, the direct deposition of nanoparticles from a local bubble to the cell membrane under the influence of ultrasound (termed as 'sonoprinting') is more likely to be the mechanism of large molecule uptake[122].

\subsection{Ultrasound sensitive substances: Ultrasound Contrast Agents (UCAs):}

The use of microbubbles as UCA in vascular imaging is well established[123] and has helped to address the impending need to resolve the relatively low resolution of traditional ultrasound imaging[124]. UCAs play an important role in contrast-enhanced ultrasonography (CEUS) by acting as preferential scatterers of sound and thus enhancing the returning ultrasound signal from highlyperfused regions of tissue[125]. Alongside imaging enhancement applications such as tumor identification[126128], myocardial perfusion[129], and vessel patency assessment[130, 131]. UCAs have also proven to be effective in therapeutic applications such as drug delivery[132, 133], hyperthermia treatment[134-136] and BBB opening[137, 138].

The first generation of UCA consisted of an air-filled microbubble encapsulated by an albumin shell[139]. Second generation UCAs contain high-molecular-weight gases such as PFCs $\left(\mathrm{C}_{3} \mathrm{~F}_{8}, \mathrm{C}_{4} \mathrm{~F}_{10}\right.$, etc.) and sulfur hexafluoride $\left(\mathrm{SF}_{6}\right)$, which have lower solubility in blood and thus increase the lifetime of the microbubbles in circulation. The shell could be albumin, phospholipid, polymeric or a combination of these materials[140, 141]. The size of clinically approved UCAs is on the order of several microns and is small in comparison with the wavelength of diagnostic ultrasound 
(order $1 \mathrm{~mm}$ ), meaning that bubbles are symmetrically driven and undergo volumetric oscillations in the absence of nearby boundaries or bubbles. The low density and high compressibility of the gas core in UCAs can enable efficient ultrasound scattering, thus significantly increase the backscattered signal emanating from bubble-perfused areas[142].

By further modifying UCAs with targeting ligands (antibodies, carbohydrates, peptides, etc.), the imaging and therapeutic application of UCAs could extend to the molecular level. Active targeting can be achieved by incorporating antibodies, carbohydrates or peptides onto the surface of UCAs[143]. The binding process begins when the ligand on the bubble surface contacts specific receptors on the vascular wall or cell surface, and will hold as long as the strength of binding outweighs the shearing effects of surrounding fluid flow (blood flow in most cases)[144]. Note that, as has been discussed in section 4.1, UCAs have the potential of becoming a next-generation gene/drug delivery vehicle, it is highly likely that targeting capabilities of UCAs could lead to the development of more effective and controllable on-site delivery platforms.

The acoustic response of UCAs differs for different formulations[145]. The relationship between UCA formulation and two vital acoustic characteristics, -frequency-dependent acoustic scattering and proclivity for inducing cavitation -- has been widely explored due to their importance in diagnostic and therapeutic applications. Changes in linear bubble dynamics (resonance frequency and acoustic damping) can be attributed to the varying viscosities and interfacial tensions of different encapsulating shells[146148]. The nonlinear response of UCAs has also been reported to be dependent on physical properties of UCA's. Guo et al. measured the inertial cavitation threshold pressures and frequency-dependent acoustic attenuation of SonoVue (Bracco Diagnostics Inc., Geneva, Switzerland) and KangRun (KangRun Pharmaceutical Co., Hunan, China) in vitro, and proposed that the smaller dilatational viscosity and shell interfacial tension might account for the lower IC threshold of SonoVue[149].

Despite their widely recognized imaging enhancement abilities, UCAs consisting of stabilized bubbles on the order of micrometers suffer from relatively short circulation time and insufficient accumulation in tumor areas in comparison with those of nanoscale particles[150]. Researchers have proposed the substitution of the gas core with a superheated liquid PFC (dodecafluoropentane, PFH, perfluoroheptane, etc.) to form much smaller nanodroplets[151]. Due to much smaller size, liquid nanodroplets can potentially penetrate the endothelial gap in tumor vasculature, passively accumulate in tumor regions and thus prolong circulation time[115, 140]. To exhibit ultrasound enhancement ability, nanodroplets are required to undergo phase-transition from liquid into gaseous state. As is illustrated in Figure 4, such a phase transition can be initiated by heating and/or imposing a transient negative pressure state (associated with the rarefaction portion of ultrasonic forcing) which suppresses the boiling temperature and enhances the likelihood of a phase change. For a particular core composition, the boiling temperature of an encapsulated nanodroplet is elevated due to the Laplace pressure induced by the interfacial tension of the encapsulating shell[150]. The stability of nanodroplets has been shown to decrease with the lowering boiling point of encapsulated core. When placed under ultrasound irradiation, nanodroplets containing a lower boiling point core require less imposed acoustic rarefaction to trigger vaporization[152].

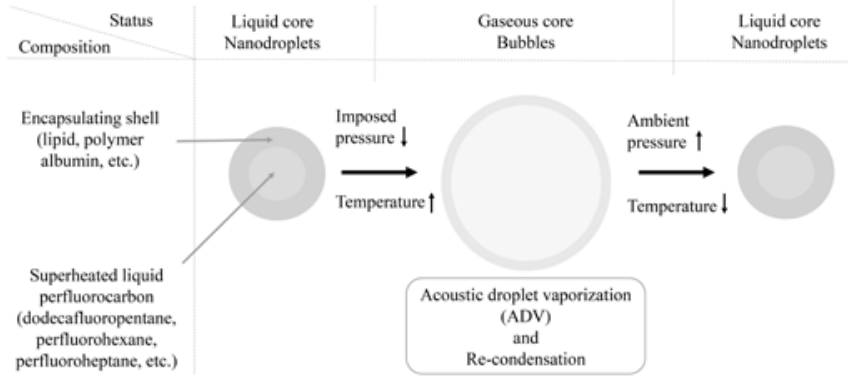

Fig. 4. Illustration of nanodroplet response to temperature and pressure variation. Imposed pressure variation could be caused by either ambient pressure or acoustic pressure. [150]

The droplet behavior after ultrasound activation is also dependent on core composition. Sheeran et al. reported a unique overexpansion characteristic of droplets with a volatile PFC core[153]. The droplet-to-bubble expansion process of contrast agents possessing cores of (a) decafluorobutane (DFB, boiling point $-2^{\circ} \mathrm{C}$ ) and (b) octafluoropropane (OFP, boiling point $-36.7^{\circ} \mathrm{C}$ ), exposed to a 2 cycle pulse of $8 \mathrm{MHz}$ ultrasound, are shown in Figure 5. As DFB and OFB are relatively volatile compounds, these droplets underwent a rapid expansion to a maximum radius followed by a damped oscillation, culminating in a new equilibrium size[153]. The ultrasound input required for vaporization has also been reported to increase with decreasing droplet size (greater Laplace pressure to work against) and increasing ultrasound frequency (less time for the phase transition to occur while under tension)[151, 154]. This acoustically induced phase transition process is often termed as acoustic droplet vaporization (ADV), and a variety of effects such as vascular occlusion[155], transcranial ultrasound phase aberration[156], ablation margin estimation[157] as well as inertial cavitation[158] can be realized when employing appropriate ultrasound parameters.

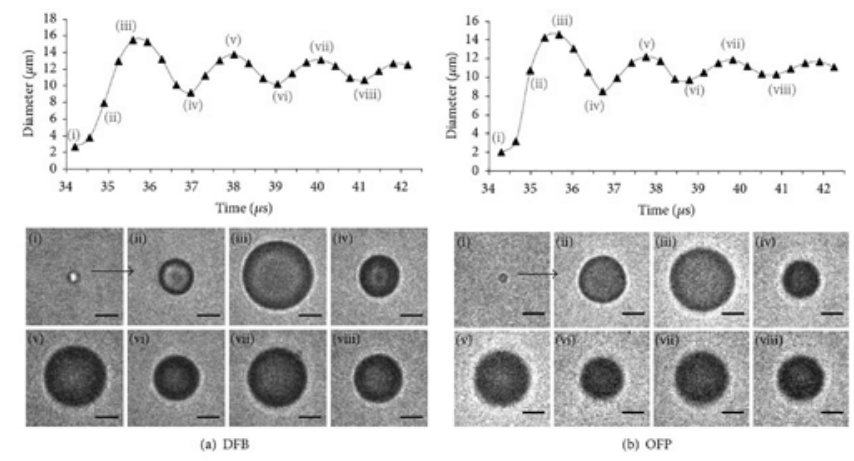

Fig. 5. Droplet vaporization and expansion process after activation by 2 cycles of $8 \mathrm{MHz}$ ultrasound. The scale bar represents $5 \mu \mathrm{m}$. From Sheeran et al [153]. Copyright (C) 
Institute of Physics and Engineering in Medicine. Reproduced by permission of IOP Publishing. All rights reserved.

\section{SONO-PHOTODYNAMIC THERAPY (SPDT)}

As some sensitizers appears to be sensitive to both ultrasound and light, the idea of combining SDT with PDT comes very naturally; after the administration of appropriate sensitizers, PDT and SDT can be performed successively (the treatment order may or may not matter). In 2000, Jin et al. performed SPDT on skin squamous cell carcinoma bearing $\mathrm{C} 3 \mathrm{H} / \mathrm{HeN}$ mice using either pheophorbide Aderivative PH-1126 or ATX-70[12]. The survival period of mice treated with combined therapies was significantly prolonged for both sensitizers, and even reached 88\% survival 120 days post-treatment with PH-1126 treated group. The synergetic inhibition effect of PDT and SDT suggest that sensitizers can be applied at half the regular dose/concentration with SPDT, which would lessen the photosensitivity brought about by PSs[12].

Similar promotion in therapeutic efficacy was found with Chlorin e6 in glioma C6, where the area of necrosis in tumor tissues caused by SPDT was found $25-30 \%$ larger than that of individual PDT/SDT treatment[13]. Sinoporphyrin sodium assisted SPDT was successfully performed on 4T1implanted mice, showing cavitational effects during the process[24]. Miyoshi et al. exhibited tumor inhibition ability with 5- aminolevulinic acid (5-ALA) and $\mathrm{TiO}_{2}$ nanoparticles in $\mathrm{C} 3 \mathrm{H} / \mathrm{HeN}$ mouse bearing skin squamous cell carcinoma[159]. In an animal study where sonnelux served as the sono-photosensitizer, El-Kaream et al. reported that pulsed ultrasound wave showed higher therapeutic efficacy than continuous ultrasound wave in the presences of synthesized sensitizer. By adding infrared laser to the treatment, tumor volume can be further reduced[160].

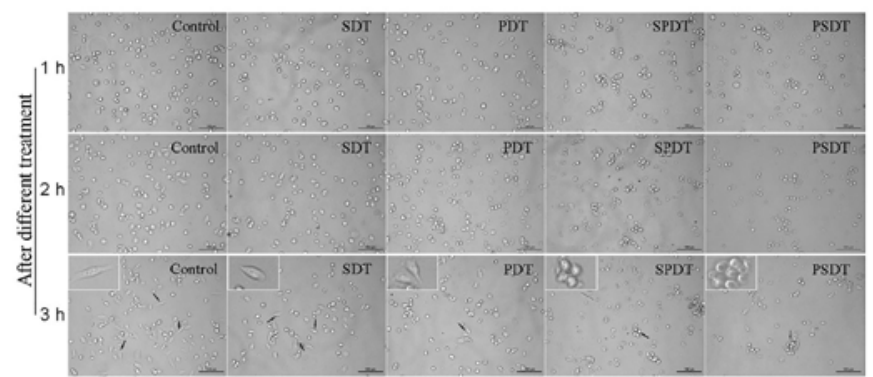

Fig. 6. Comparison of cell adhesion conditions at different times post-treatment with different treatment methods. The scale bar represents $100 \mu \mathrm{m}$, and the black arrows highlight typical cell morphology which is shown in the upper left corner of each figure. The ability for cells to adhere is modified in different ways by different treatment protocols. From Wang et al [162]. Copyright (c) Astro Ltd. Reproduced by permission of IOP Publishing. All rights reserved.

Alongside direct tumor inhibition, SPDT was also reported to elicit anti-metastatic and pro-apoptotic effects on breast cancer using Ce6 as a sensitizer[161]. A drop in cell mobility was also observed in SPDT-treated MDA-MB-231 cells[162]. Figure 6 shows representative images of MDA-
MB-231 cell morphology at various time points $(1,2$ and 3 hours) after different treatment protocols (SDT, PDT, SPDT and PSDT). After being re-seeded into culture dish after treatment, pictures of cells were taken to show the degree of cell adhesion. It can be seen from the figure that cells in the control group began to adhere to the culture dish 3 hours post-seeding, while cells in the SDT $\left(0.36 \mathrm{~W} \mathrm{~cm}{ }^{-2}\right.$ ultrasound, 1-minute duration) and PDT (1.2 $\mathrm{J} \mathrm{cm}^{-2}$ laser, 2minute duration) groups showed moderate adhesion, and cells in the SPDT $\left(0.36 \mathrm{~W} \mathrm{~cm}^{-2}\right.$ ultrasound, 1-minute duration followed by $1.2 \mathrm{~J} \mathrm{~cm}^{-2}$ laser, 2-minute duration) and PSDT (1.2 J cm${ }^{-2}$ laser, 2-minute duration followed by 0.36 $\mathrm{W} \mathrm{cm}{ }^{-2}$ ultrasound, 1-minute duration) groups showed few adhesions. When the order of PDT and SDT application was altered, cell viability results and tumor inhibition effects showed no significant difference[161]. However, as ultrasound irradiation could enhance cell membrane permeability, the pre-PDT SDT might be able to trigger sensitizer entry into tumor cells, thus increasing SPDT efficacy[163].

Recently, a rose bengal derivative was proposed, with the aim of improving biocompatibility and amphiphilicity[164]. A nano-theranostic platform consisting of PLGA, indocyanine green and $\mathrm{Hp}$ was designed for simultaneous NIR fluorescence imaging and SPDT[165].

In comparison with laser irradiation, the benefit of ultrasound lies in its deep penetration depth and superior focusing ability. The incorporation of ultrasound into traditional PDT was found to enhance the depth of tumor necrosis by 2-3 times in vivo, which helps overcome the limitations imposed by the rapid absorption and scattering of light in tissue[12]. Considering that SPDT shows a stronger therapeutic effect, a decrease in the dosage of sensitizer and laser/ultrasound input could be achieved using SPDT, which in turn could help in shielding peripheral normal tissues from undesired damage[24]. With the additional help of tumorspecific sensitizer or delivery platforms, more precise control over treatment area and therapeutic outcome could be realized using SPDT[161, 165].

\section{CONCLUSION AND FUTURE PERSPECTIVES}

Light and sound are two forms of energy that have played a critical role in modern medicine by enabling minimally-invasive therapies, such as PDT and SDT. However, there are persistent obstacles to the widespread applications of PDT and SDT in the clinic. Treatment depth using light is limited due to high levels of scattering and absorption. High intensity ultrasound can also result in deleterious tissue heating and suffers from beam distortion due to sound speed and density aberrations in biological tissues, particularly bone. While researchers on both sides are working to address these technology barriers, it has become very clear that the introduction of nano/micro sized sensitizers could be the solution, for they enhance sensitivity to optical/acoustical perturbations and provide spatial specificity. Therefore, by the introducing sensitizers, the required incident energy can be lowered and the treatment process can be better controlled in both space and time, thus enhancing therapeutic efficacy and safety. With new advances in nanotechnology, sensitizers can be easily modified, providing the opportunity of therapy fusion. A 
fertile ground for future research could be the combination of light and sound sensitive substances yielding "multi-mode" sensitizers for which the thresholds for both light and soundinduced therapeutic effects are lowered, providing efficient yet safe outcomes for a broader spectrum of therapies.

\section{ACKNOWLEDGMENTS}

Juan Tu and Dong Zhang designed the structure of this article. Yanye Yang and Dongxin Yang drafted and revised this article. Jason L. Raymond and Ronald A. Roy provided academical and verbal suggestions to this article.

This work was supported by the National Natural Science Foundation of China (Grant Nos. 81627802, 11474001, 11474161, 81527803, 81420108018, 11774166, 11774168 and 11674173), QingLan Project and the the Fundamental Research Funds for the Central

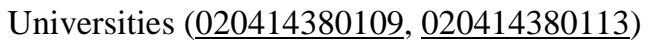

\section{CONFLICT OF INTEREST}

The authors declare no conflict of interest.

\section{LIST OF ABBREVIATIONS}

$\begin{array}{ll}\text { 5-ALA } & \text { 5- Aminolevulinic Acid } \\ \text { ADV } & \text { Acoustic Droplet Vaporization } \\ \text { BBB } & \text { Blood-Brain-Barrier } \\ \text { CEUS } & \text { Contrast-Enhanced Ultrasonography } \\ \text { DOX } & \text { Doxorubicin } \\ \text { HIFU } & \text { High Intensity Focused Ultrasound } \\ \text { HMME } & \text { Hematoporphyrin Monomethylethe } \\ \text { Hp } & \text { Hematoporphyrin } \\ \text { HSA } & \text { Human Serum Albumin } \\ \text { HSP } & \text { Heat Shock Protein } \\ \text { ISC } & \text { Intersystem Crossing } \\ \text { MRI } & \text { Magnetic Resonance Imaging } \\ \text { NIR } & \text { Near Infrared } \\ \text { PA } & \text { Photoacoustic } \\ \text { Pc } & \text { Phthalocyanines } \\ \text { PDT } & \text { Photodynamic Therapy } \\ \text { PFC } & \text { Perfluorocarbon } \\ \text { PFH } & \text { Perfluorohexane } \\ \text { PFOB } & \text { Perfluorooctyl Bromide } \\ \text { PLGA } & \text { Poly (DLlactic-co-glycolic acid) } \\ \text { PpIX } & \text { Protoporphyrin IX } \\ \text { PS } & \text { Photosensitizer } \\ \text { PTT } & \text { Photothermal Therapy } \\ \text { ROS } & \text { Reactive Oxygen Species } \\ \text { RT } & \text { Radiotherapy } \\ \text { SDT } & \text { Sonodynamic Therapy } \\ \text { SF6 } & \text { Sulfur Hexafluoride } \\ \text { SOG } & \text { Singlet Oxygen Generator } \\ \text { SPDT } & \text { Sono-photodynamic Therapy } \\ \text { UCA } & \text { Upconversion luminescence } \\ \text { UCL } & \\ \text { UCNP } & \end{array}$

\section{REFERENCES}

[1] Kennedy J. High-intensity focused ultrasound in the treatment of solid tumours. Nature Reviews Cancer, 2005; (5):321-327.

[2] Nikfarjam M, Christophi C. Interstitial laser thermotherapy for liver tumours. Br J Surg, 2003; (90):10331047.

[3] Lismont M, Dreesen L, Wuttke S. Metal-Organic Framework Nanoparticles in Photodynamic Therapy: Current Status and Perspectives. Advanced Functional Materials, 2017; (27):1606314.

[4] Dolmans D, Fukumura D, Jain R. Photodynamic therapy for cancer Nat Rev Cancer, 2003; (3):380-387.

[5] Nesi-Reis V, Lera-Nonose D, Oyama $\mathrm{J}$ et al. Contribution of Photodynamic Therapy in Wound Healing: A Systematic Review. Photodiagnosis Photodyn Ther, 2017. [6] Wen X, Li Y, Hamblin MR. Photodynamic therapy in dermatology beyond non-melanoma cancer: An update. Photodiagnosis Photodyn Ther, 2017; (19):140-152.

[7] Bashkatov AN, Genina EA, Kochubey VI, Tuchin VV. Optical properties of human skin, subcutaneous and mucous tissues in the wavelength range from 400 to $2000 \mathrm{~nm}$. Journal of Physics D: Applied Physics, 2005; (38):25432555.

[8] Mironova KE, Proshkina GM, Ryabova AV et al. Genetically Encoded Immunophotosensitizer 4D5scFvminiSOG is a Highly Selective Agent for Targeted Photokilling of Tumor Cells in Vitro. Theranostics, 2013; (3):831-840.

[9] Grebenik EA, Kostyuk AB, Deyev SM. Upconversion nanoparticles and their hybrid assemblies for biomedical applications. Russian Chemical Reviews, 2016; (85):12771296.

[10] Yumita NN, R; Umemura, K. Hematoporphyrin as a Sensitizer of Cell-damaging Effect of Ultrasound. Japanese Journal of Cancer Research, 1989; (80):319-222.

[11] Yumita NN, R; Umemura, K; Umemura, S. Synergistic Effect of Ultrasound and Hematoporphyrin on Sarcoma 180. Japanese Journal of Cancer Research, 1990; (81):304-308.

[12] Jin $\mathrm{ZH} \mathrm{MN}$, Ishiguro $\mathrm{K}$. Combination Effect of Photodynamic and Sonodynamic Therapy on Experimental Skin Squamous Cell Carcinoma in C3H/HeN Mice. Journal of Dermatology, 2000; (27):294-306.

[13] Tserkovsky DAA, E. N.; Chalau, V. N. Effects of Combined Sonodynamic and Photodynamic

Therapies with Photolon on a Glioma C6 Tumor Model. Experimental Oncology, 2012; (34):332-335.

[14] Sirsi SR, Borden MA. Advances in ultrasound mediated gene therapy using microbubble contrast agents. Theranostics, 2012; (2):1208-1222.

[15] Wu Y, Lu CT, Li WF et al. Preparation and antitumor activity of bFGF-mediated active targeting doxorubicin microbubbles. Drug Dev Ind Pharm, 2013; (39):1712-1719. [16] Abrahamse H, Hamblin MR. New photosensitizers for photodynamic therapy. Biochem J, 2016; (473):347-364.

[17] van Straten D, Mashayekhi V, de Bruijn $\mathrm{H}$ et al. Oncologic Photodynamic Therapy: Basic Principles, Current Clinical Status and Future Directions. Cancers, 2017; (9):19. 
[18] Castano AP, Demidova TN, Hamblin MR. Mechanisms in photodynamic therapy: part one-photosensitizers, photochemistry and cellular localization. Photodiagnosis and Photodynamic Therapy, 2004; (1):279-293.

[19] Castano AP, Demidova TN, Hamblin MR. Mechanisms in photodynamic therapy: part two-cellular signaling, cell metabolism and modes of cell death. Photodiagnosis and Photodynamic Therapy, 2005; (2):1-23.

[20] Lafond M, Yoshizawa S, Umemura SI. Sonodynamic Therapy: Advances and Challenges in Clinical Translation. J Ultrasound Med, 2018.

[21] Pan X, Wang H, Wang S et al. Sonodynamic therapy (SDT): a novel strategy for cancer nanotheranostics. Sci China Life Sci, 2018; (61):415-426.

[22] Rengeng L, Qianyu Z, Yuehong L et al. Sonodynamic therapy, a treatment developing from photodynamic therapy. Photodiagnosis Photodyn Ther, 2017; (19):159-166.

[23] Wang X, Jia Y, Wang P et al. Current status and future perspectives of sonodynamic therapy in glioma treatment. Ultrasonics Sonochemistry, 2017; (37):592-599.

[24] Liu Y, Wang P, Liu Q, Wang X. Sinoporphyrin sodium triggered sono-photodynamic effects on breast cancer both in vitro and in vivo. Ultrason Sonochem, 2016; (31):437-448.

[25] Saito M IT, Nagayama D. Photodynamic therapy with verteporfin for age-related macular degeneration or polypoidal choroidal vasculopathy: comparison of the presence of serous retinal pigment epithelial detachment. British Journal of Ophthalmology, 2008; (92).

[26] Shavkuta BS, Gerasimov MY, Minaev NV et al. Highly effective $525 \mathrm{~nm}$ femtosecond laser crosslinking of collagen and strengthening of a human donor cornea. Laser Physics Letters, 2018; (15):015602.

[27] Robertson CA, Evans DH, Abrahamse $H$. Photodynamic therapy (PDT): a short review on cellular mechanisms and cancer research applications for PDT. J Photochem Photobiol B, 2009; (96):1-8.

[28] Juzeniene A, Moan J. The history of PDT in Norway Part one: Identification of basic mechanisms of general PDT. Photodiagnosis Photodyn Ther, 2007; (4):3-11.

[29] Agostinis PB, E; Breyssens, H. Regulatory pathways in photodynamic therapy induced apoptosis. In: 10th Congress of the European-Society-for-Photobiology. Vienna, AUSTRIA: Photochemical \& Photobiological Sciences 2004. pp. 721-729.

[30] Li X, Kolemen S, Yoon J, Akkaya EU. Activatable Photosensitizers: Agents for Selective Photodynamic Therapy. Advanced Functional Materials, 2017; (27):1604053.

[31] Fan W, Huang P, Chen X. Overcoming the Achilles' heel of photodynamic therapy. Chem Soc Rev, 2016; (45):6488-6519.

[32] Tian J ZJ, Shen Z. A pH-activatable and anilinesubstituted photosensitizer for near-infrared cancer theranostics. Chemical Science, 2015; (6):5969-5977.

[33] Battogtokh GK, YT. Active-targeted pH-responsive albumin-photosensitizer conjugate nanoparticles as theranostic agents. Journal of Materials Chemistry B, 2015; (3):9349-9359.

[34] Cottrell WJ, Paquette AD, Keymel KR et al. Irradiancedependent photobleaching and pain in delta-aminolevulinic acid-photodynamic therapy of superficial basal cell carcinomas. Clin Cancer Res, 2008; (14):4475-4483.

[35] Foster TM, RS; Bryant, RG; Knox, RS; Gibson, SL; Hilf, R. Oxygen Consumption and Diffusion Effect in Photodynamic Therapy. Radiation Research, 1991; (126):296-303.

[36] Wang W, Moriyama LT, Bagnato VS. Photodynamic therapy induced vascular damage: an overview of experimental PDT. Laser Physics Letters, 2013; (10):023001. [37] Sitnik TH, BW. The Effect of Fluence Rate on Tumor and Normal Tissue Responses to Photodynamic Therapy. Photochemistry and Photobiology, 1998; (67):462-466.

[38] Sitnik TH, JA; Henderson, BW Reduction of Tumour Oxygenation During and After Photodynamic Therapy in vivo: Effects of Fluence Rate. British Journal of Cancer, 1998; (77):1386-1394.

[39] Song. C. W. SA, Osborn J. L., \& Iwata K. Tumour oxygenation is increased by hyperthermia at mild temperatures. International Journal of Hyperthermia, 2009; (25):91-95.

[40] Bolfarini GC, Siqueira-Moura MP, Demets GJF et al. In vitro evaluation of combined hyperthermia and photodynamic effects using magnetoliposomes loaded with cucurbituril zinc phthalocyanine complex on melanoma. Journal of Photochemistry and Photobiology B: Biology, 2012; (115):1-4.

[41] Di Corato RB, G; Kolosnjaj-Tabi, J; Espinosa, A ; Clement, O ; Silva, AKA; Menager, C ; Wilhelm, C Combining Magnetic Hyperthermia and Photodynamic Therapy for Tumor Ablation with Photoresponsive Magnetic Liposomes. ACS Nano, 2015; (9):2904-2916.

[42] Matzi V, Maier A, Sankin O et al. Photodynamic therapy enhanced by hyperbaric oxygenation in palliation of malignant pleural mesothelioma: clinical experience. Photodiagnosis and Photodynamic Therapy, 2004; (1):57-64. [43] Chen Q, Huang Z, Chen H et al. Improvement of Tumor Response by Manipulation of Tumor Oxygenation During Photodynamic Therapy. Photochemistry and Photobiology, 2002; (76):197-203.

[44] Day RA, Estabrook DA, Logan JK, Sletten EM. Fluorous photosensitizers enhance photodynamic therapy with perfluorocarbon nanoemulsions. Chem Commun (Camb), 2017; (53):13043-13046.

[45] Cheng Y, Cheng H, Jiang C et al. Perfluorocarbon nanoparticles enhance reactive oxygen levels and tumour growth inhibition in photodynamic therapy. Nat Commun, 2015; (6):8785.

[46] Clark L, Gollan F. Survival of Mammals Breathing Organic Liquids Equilibrated with Oxygen at Atmospheric Pressure. Science, 1966; (152).

[47] Castro CI, Briceno JC. Perfluorocarbon-based oxygen carriers: review of products and trials. Artif Organs, 2010; (34):622-634.

[48] Jahr J, Walker V, Manoochehri K. Blood substitutes as pharmacotherapies in clinical practice. Current Opinion in Anaesthesiology, 2007; (20):325-330.

[49] Fingar VH MT, Henderson BW. Modification of Photodynamic Therapy-induced Hypoxia by Fluosol-DA (20\%) and Carbogen Breathing in Mice. Cancer Res, 1988; (48):3350-3354. 
[50] Wang YG, Kim H, Mun S et al. Indocyanine greenloaded perfluorocarbon nanoemulsions for bimodal (19)Fmagnetic resonance/nearinfrared fluorescence imaging and subsequent phototherapy. Quant Imaging Med Surg, 2013; (3):132-140

[51] Ren H, Liu J, Su F et al. Relighting Photosensitizers by Synergistic Integration of Albumin and Perfluorocarbon for Enhanced Photodynamic Therapy. ACS Appl Mater Interfaces, 2017; (9):3463-3473.

[52] Sheng D, Liu T, Deng L et al. Perfluorooctyl bromide \& indocyanine green co-loaded nanoliposomes for enhanced multimodal imaging-guided phototherapy. Biomaterials, 2018; (165):1-13.

[53] Wang J, Liu L, You Q et al. All-in-One Theranostic Nanoplatform Based on Hollow MoSx for Photothermallymaneuvered Oxygen Self-enriched Photodynamic Therapy. Theranostics, 2018; (8):955-971.

[54] Song X, Feng L, Liang C et al. Ultrasound Triggered Tumor Oxygenation with Oxygen-Shuttle Nanoperfluorocarbon to Overcome Hypoxia-Associated Resistance in Cancer Therapies. Nano Lett, 2016; (16):61456153.

[55] Chen H, Zhou X, Gao Y et al. Recent progress in development of new sonosensitizers for sonodynamic cancer therapy. Drug Discov Today, 2014; (19):502-509.

[56] Tachibana K, Feril LB, Jr., Ikeda-Dantsuji Y. Sonodynamic therapy. Ultrasonics, 2008; (48):253-259.

[57] Rosenthal I, Sostaric JZ, Riesz P. Sonodynamic therapy--a review of the synergistic effects of drugs and ultrasound. Ultrason Sonochem, 2004; (11):349-363.

[58] Hirschberg H, Madsen S. Synergistic efficacy of ultrasound, sonosensitizers and chemotherapy: a review. Therapeutic Delivery, 2017; (8):331-342.

[59] Chen J, Luo H, Liu Y et al. Oxygen-Self-Produced Nanoplatform for Relieving Hypoxia and Breaking Resistance to Sonodynamic Treatment of Pancreatic Cancer. ACS Nano, 2017; (11):12849-12862.

[60] Ju D, Yamaguchi F, Zhan G et al. Hyperthermotherapy enhances antitumor effect of 5-aminolevulinic acid-mediated sonodynamic therapy with activation of caspase-dependent apoptotic pathway in human glioma. Tumour Biol, 2016; (37):10415-10426.

[61] Umemura SY, N; Nishigaki, R; Umemura, K. Mechanism of Cell Damage by Ultrasound in Combination with Hematoporphyrin. Japanese Journal of Cancer Research, 1990; (81):962-966.

[62] Kessel D. JR, Fowlkes J.B. \& Cain C. Porphyrininduced Enhancement of Ultrasound Cytotoxicity. International Journal of Radiation Biology, 1994; (66): 221228.

[63] Tang $\mathrm{W}$, Liu Q, Zhang $\mathrm{J}$ et al. In vitro activation of mitochondria-caspase signaling pathway in sonodynamic therapy-induced apoptosis in sarcoma 180 cells. Ultrasonics, 2010; (50):567-576.

[64] Li JH, Song DY, Xu YG et al. In vitro study of haematoporphyrin monomethyl ether-mediated sonodynamic effects on C6 glioma cells. Neurol Sci, 2008; (29):229-235. [65] Su X, Wang P, Wang X et al. Apoptosis of U937 cells induced by hematoporphyrin monomethyl ether-mediated sonodynamic action. Cancer Biother Radiopharm, 2013; (28):207-217.

[66] Feng Q, Zhang W, Yang X et al. pH/Ultrasound DualResponsive Gas Generator for Ultrasound Imaging-Guided Therapeutic Inertial Cavitation and Sonodynamic Therapy. Adv Healthc Mater, 2017.

[67] Yan S, Lu M, Ding X et al. HematoPorphyrin Monomethyl Ether polymer contrast agent for ultrasound/photoacoustic dual-modality imaging-guided synergistic high intensity focused ultrasound (HIFU) therapy. Sci Rep, 2016; (6).

[68] Su X WX, Zhang K, Yang S , Liu Q, Leung AW , Xu C , Wang P Sonodynamic therapy induces apoptosis of human leukemia HL-60 cells in the presence of protoporphyrin IX. General Physiology and Biophysics, 2016; (35):155-164.

[69] Huang P, Qian X, Chen Y et al. MetalloporphyrinEncapsulated Biodegradable Nanosystems for Highly Efficient Magnetic Resonance Imaging-Guided Sonodynamic Cancer Therapy. J Am Chem Soc, 2017; (139):1275-1284.

[70] Umemura KY, N; Nishigaki, R; Umemura, Si. Sonodynamically induced antitumor effect of pheophorbide a. Cancer Letters, 1996; (102):151-157.

[71] Xu ZY, Wang K, Li XQ et al. The ABCG2 transporter is a key molecular determinant of the efficacy of sonodynamic therapy with Photofrin in glioma stem-like cells. Ultrasonics, 2013; (53):232-238.

[72] Yumita NN, R; Umemura, S. Sonodynamically induced antitumor effect of Photofrin II on colon 26 carcinoma. Journal of Cancer Research and Clinical Oncology, 2000; (126):601-606.

[73] Tachibana K. KN, Okumura M., Eguchi H., Tachibana S. Enhancement of cell killing of HL-60 cells by ultrasound in the presence of the photosensitizing drug Photofrin II. Cancer Letters, 1993; (72):195-199.

[74] Yumita N, Okudaira K, Momose Y, Umemura S. Sonodynamically induced apoptosis and active oxygen generation by gallium-porphyrin complex, ATX-70. Cancer Chemother Pharmacol, 2010; (66):1071-1078.

[75] Umemura Si; Yumita NN, R. Enhancement of Ultrasonically Induced Cell Damage by a Gallium-porphyrin Complex, ATX-70. Japanese Journal of Cancer Research, 1993; (84):582-588.

[76] Abe HK, M; Tachibana, K. Targeted sonodynamic therapy of cancer using a photosensitizer conjugated with antibody against carcinoembryonic antigen. Anticancer Research, 2002; (22):1575-1580.

[77] Yumita NN, R; Sakata, I. Sonodynamically Induced Antitumor Effect of 4-Formyloximethylidene-3-hydroxy-2vinyl-deuterio-porphynyl(IX)-6,7-diaspartic Acid (ATXS10). Japanese Journal of Cancer Research, 2000; (91):255260

[78] Yumita N, Sakata I, Nakajima S, Umemura S-i. Ultrasonically induced cell damage and active oxygen generation by 4-formyloximeetylidene-3-hydroxyl-2-vinyldeuterio-porphynyl(IX)-6-7-diaspartic acid: on the mechanism of sonodynamic activation. Biochimica et Biophysica Acta (BBA) - General Subjects, 2003; (1620):179-184. 
[79] Yumita N, Han QS, Kitazumi I, Umemura S. Sonodynamically-induced apoptosis, necrosis, and active oxygen generation by mono-l-aspartyl chlorin e6. Cancer Sci, 2008; (99):166-172.

[80] Yumita N, Iwase $\mathrm{Y}$, Nishi $\mathrm{K}$ et al. Involvement of reactive oxygen species in sonodynamically induced apoptosis using a novel porphyrin derivative. Theranostics, 2012; (2):880-888.

[81] Hachimine $\mathrm{K}$, Shibaguchi $\mathrm{H}$, Kuroki $\mathrm{M}$ et al. Sonodynamic therapy of cancer using a novel porphyrin derivative, DCPH-P-Na(I), which is devoid of photosensitivity. Cancer Sci, 2007; (98):916-920.

[82] Yumita NK, K; Sasaki, K. Sonodynamic effect of erythrosin B on sarcoma 180 cells in vitro. Ultrasonics Sonochemistry, 2002; (9):259-265.

[83] Umemura SY, N; Umemura, K; Nishigaki, R. Sonodynamically induced effect of rose bengal on isolated sarcoma 180 cells. Cancer Chemother Pharmacol, 1999; (43):389-393.

[84] Nonaka MY, Masaaki; Yoshino, Shinichiro. Sonodynamic Therapy Consisting of Focused Ultrasound and a Photosensitizer Causes a Selective Antitumor Effect in a Rat Intracranial Glioma Model. In: 11th Annual Meeting of the Society-of-Biotherapeutic-Approaches Tokyo, JAPAN: Anticancer Research; 2009. pp. 943-950

[85] Sugita NK, Ken-ichi; Sasaki, Kazuaki. Synthesis of Amphiphilic Derivatives of Rose Bengal and Their Tumor Accumulation. Bioconjugate chemistry, 2007; (18):866-873.

[86] Sugita NI, Yumiko; Yumita, Nagahiko. Sonodynamically Induced Cell Damage Using Rose Bengal Derivative. Anticancer Research, 2010; (30):3361-3366.

[87] Chen Z, Li J, Song $\mathrm{X}$ et al. Use of a novel sonosensitizer in sonodynamic therapy of U251 glioma cells in vitro. Exp Ther Med, 2012; (3):273-278.

[88] Sviridov AP, Andreev VG, Ivanova EM et al. Porous silicon nanoparticles as sensitizers for ultrasonic hyperthermia. Applied physics letters, 2013; (103):193110.

[89] Yumita N WT, Chen FS. Induction of Apoptosis by Functionalized Fullerene-based Sonodynamic Therapy in HL-60 cells. Anticancer Research, 2016; (36):2665-2674.

[90] Qian J, Gao Q. Sonodynamic Therapy Mediated by Emodin Induces the Oxidation of Microtubules to Facilitate the Sonodynamic Effect. Ultrasound Med Biol, 2018; (44):853-860.

[91] Gao Q, Wang F, Guo S et al. Sonodynamic effect of an anti-inflammatory agent--emodin on macrophages. Ultrasound Med Biol, 2011; (37):1478-1485.

[92] Qian X, Zheng Y, Chen Y. Micro/NanoparticleAugmented Sonodynamic Therapy (SDT): Breaking the Depth Shallow of Photoactivation. Adv Mater, 2016; (28):8097-8129.

[93] Harada Y, Ogawa K, Irie Y et al. Ultrasound activation of TiO2 in melanoma tumors. J Control Release, 2011; (149):190-195.

[94] Yamaguchi S, Kobayashi $\mathrm{H}$, Narita $\mathrm{T}$ et al. Sonodynamic therapy using water-dispersed TiO2polyethylene glycol compound on glioma cells: comparison of cytotoxic mechanism with photodynamic therapy. Ultrason Sonochem, 2011; (18):1197-1204.
[95] Shen S, Wu L, Liu J et al. Core-shell structured Fe3O4@TiO2-doxorubicin nanoparticles for targeted chemo-sonodynamic therapy of cancer. Int J Pharm, 2015; (486):380-388.

[96] Shen S, Guo X, Wu L et al. Dual-core@shell-structured Fe3O4-NaYF4@TiO2 nanocomposites as a magnetic targeting drug carrier for bioimaging and combined chemosonodynamic therapy. J. Mater. Chem. B, 2014; (2):57755784.

[97] Lentacker I, De Cock I, Deckers R et al. Understanding ultrasound induced sonoporation: definitions and underlying mechanisms. Adv Drug Deliv Rev, 2014; (72):49-64.

[98] Ward M, Wu J, Chiu J-F. Ultrasound-induced cell lysis and sonoporation enhanced by contrast agents. J Acoust Soc Am, 1999; (105):2951-2957.

[99] Lakshmanan S, Gupta GK, Avci P et al. Physical energy for drug delivery; poration, concentration and activation. Adv Drug Deliv Rev, 2014; (71):98-114.

[100] Wu J, Nyborg WL. Ultrasound, cavitation bubbles and their interaction with cells. Adv Drug Deliv Rev, 2008; (60):1103-1116.

[101] Kooiman K, Foppen-Harteveld M, van der Steen AF, de Jong N. Sonoporation of endothelial cells by vibrating targeted microbubbles. J Control Release, 2011; (154):35-41. [102] Fan ZL, HY ; Mayer, M ; Deng, CX Spatiotemporally controlled single cell sonoporation. Proceedings of the National Academy of Sciences of the United States of America, 2012; (109):16486-16491.

[103] van Wamel A, Kooiman K, Harteveld $\mathrm{M}$ et al. Vibrating microbubbles poking individual cells: drug transfer into cells via sonoporation. J Control Release, 2006; (112):149-155.

[104] Fan P, Zhang Y, Guo X et al. Cell-cycle-specific Cellular Responses to Sonoporation. Theranostics, 2017; (7):4894-4908.

[105] Hu Y, Wan JM, Yu AC. Membrane perforation and recovery dynamics in microbubble-mediated sonoporation. Ultrasound Med Biol, 2013; (39):2393-2405.

[106] Spurny P, Oberst J, Heinlein D. Photographic observations of Neuschwanstein, a second meteorite from the orbit of the Pribram chondrite. Nature, 2003; (423):151153.

[107] Lauterborn W, Kurz T. Physics of bubble oscillations. Reports on Progress in Physics, 2010; (73):106501.

[108] Allen J, Roy R, Church C. On the Role of Shear Viscosity in Mediating Inertial Cavitation from Short-Pulse, Megahertz-Frequency Ultrasound. IEEE Transactions on Ultrasonics Ferroelectrics \& Frequency Control, 1997; (44):743-751.

[109] Ohl CD, Arora M, Ikink R et al. Sonoporation from jetting cavitation bubbles. Biophys J, 2006; (91):4285-4295. [110] Prentice P, Cuschieri A, Dholakia K et al. Membrane disruption by optically controlled microbubble cavitation. Nature Physics, 2005; (1):107-110.

[111] Nishikawa M, Huang L. Nonviral Vectors in the New Millennium: Delivery Barriers in Gene Transfer. Human Gene Therapy, 2001; (12):861-870.

[112] Lentacker I, De Geest B, Vandenbroucke R et al. Ultrasound-responsive polymer-coated microbubbles that bind and protect DNA. Langmuir, 2006; (22):7273-7278. 
[113] Frenkel P, Chen S, Thai T et al. DNA-loaded albumin microbubbles enhance ultrasound-mediated transfection in vitro. Ultrasound Med Biol, 2002; (28):817-822.

[114] Bekeredjian R, Chen S, Grayburn PA, Shohet RV. Augmentation of cardiac protein delivery using ultrasound targeted microbubble destruction. Ultrasound Med Biol, 2005; (31):687-691.

[115] Wilson K, Homan K, Emelianov S. Biomedical photoacoustics beyond thermal expansion using triggered nanodroplet vaporization for contrast-enhanced imaging. Nat Commun, 2012; (3):618.

[116] Teupe C. Vascular Gene Transfer of Phosphomimetic Endothelial Nitric Oxide Synthase (S1177D) Using Ultrasound-Enhanced Destruction of Plasmid-Loaded Microbubbles Improves Vasoreactivity. Circulation, 2002; (105):1104-1109.

[117] Bekeredjian R, Chen S, Frenkel PA et al. Ultrasoundtargeted microbubble destruction can repeatedly direct highly specific plasmid expression to the heart. Circulation, 2003; (108):1022-1026.

[118] Haag P, Frauscher F, Gradl J et al. Microbubbleenhanced ultrasound to deliver an antisense oligodeoxynucleotide targeting the human androgen receptor into prostate tumours. J Steroid Biochem Mol Biol, 2006; (102):103-113.

[119] Christiansen JP, French BA, Klibanov AL et al. Targeted tissue transfection with ultrasound destruction of plasmid-bearing cationic microbubbles. Ultrasound Med Biol, 2003; (29):1759-1767.

[120] Tinkov S, Coester C, Serba S et al. New doxorubicinloaded phospholipid microbubbles for targeted tumor therapy: in-vivo characterization. J Control Release, 2010; (148):368-372.

[121] Tinkov S, Winter G, Coester C, Bekeredjian R. New doxorubicin-loaded phospholipid microbubbles for targeted tumor therapy: Part I--Formulation development and in-vitro characterization. J Control Release, 2010; (143):143-150.

[122] De Cock I, Lajoinie G, Versluis M et al. Sonoprinting and the importance of microbubble loading for the ultrasound mediated cellular delivery of nanoparticles. Biomaterials, 2016; (83):294-307.

[123] Cosgrove D, Harvey C. Clinical uses of microbubbles in diagnosis and treatment. Med Biol Eng Comput, 2009; (47):813-826.

[124] Weissleder R, Mahmood U. Molecular Imaging1. Radiology, 2001; (219):316-333.

[125] Quaia E. Microbubble ultrasound contrast agents: an update. Eur Radiol, 2007; (17):1995-2008.

[126] Bartolotta TV, Vernuccio F, Taibbi A, Lagalla R. Contrast-Enhanced Ultrasound in Focal Liver Lesions: Where Do We Stand? Semin Ultrasound CT MR, 2016; (37):573-586.

[127] Wu Q, Wang Y, Li Y et al. Diagnostic value of contrast-enhanced ultrasound in solid thyroid nodules with and without enhancement. Endocrine, 2016; (53):480-488.

[128] Mori N, Mugikura S, Takahashi S et al. Quantitative Analysis of Contrast-Enhanced Ultrasound Imaging in Invasive Breast Cancer: A Novel Technique to Obtain Histopathologic Information of Microvessel Density. Ultrasound Med Biol, 2017; (43):607-614.
[129] Porter TR, Xie F. Myocardial perfusion imaging with contrast ultrasound. JACC Cardiovasc Imaging, 2010; (3):176-187.

[130] Hoffmann R, Barletta G, von Bardeleben S et al. Analysis of left ventricular volumes and function: a multicenter comparison of cardiac magnetic resonance imaging, cine ventriculography, and unenhanced and contrast-enhanced two-dimensional and three-dimensional echocardiography. J Am Soc Echocardiogr, 2014; (27):292301.

[131] Madani A, Beletsky V, Tamayo A et al. High-risk asymptomatic carotid stenosis Ulceration on 3D ultrasound vs TCD microemboli. Neurology, 2011; (77):744-750.

[132] Eisenbrey JR, Burstein OM, Kambhampati R et al. Development and optimization of a doxorubicin loaded poly(lactic acid) contrast agent for ultrasound directed drug delivery. J Control Release, 2010; (143):38-44.

[133] Cochran MC, Eisenbrey J, Ouma RO et al. Doxorubicin and paclitaxel loaded microbubbles for ultrasound triggered drug delivery. Int J Pharm, 2011; (414):161-170.

[134] Holt RG, Roy RA. Measurements of bubble-enhanced heating from focused, mhz-frequency ultrasound in a tissuemimicking material. Ultrasound Med Biol, 2001; (27):13991412.

[135] Watmough DJL, R.; Ghezzi, F.; Quan, K. M.; Watmough, J. A.; Khizhnyak, E.; Pashovkin T.N.; Sarvazyan A.P. The effect of gas bubbles on the production of ultrasound hyperthermia at $0.75 \mathrm{MHz}$ : A phantom study. Ultrasound Med Biol, 1993; (19):231-241.

[136] Li C, Zhang Y, Li Z et al. Light-Responsive Biodegradable Nanorattles for Cancer Theranostics. Adv Mater, 2018; (30).

[137] Sheikov N, McDannold N, Vykhodtseva N et al. Cellular mechanisms of the blood-brain barrier opening induced by ultrasound in presence of microbubbles. Ultrasound Med Biol, 2004; (30):979-989.

[138] Lammers T, Koczera P, Fokong S et al. Theranostic USPIO-Loaded Microbubbles for Mediating and Monitoring Blood-Brain Barrier Permeation. Adv Funct Mater, 2015; (25):36-43.

[139] Bleeker H, Shung K, Barnhart J. Ultrasonic characterization of Albunex (C) , a new contrast agent. Journal of the Acoustical Society of America, 1990; (87):1792-1797. [140] Guvener N, Appold L, de Lorenzi F et al. Recent advances in ultrasound-based diagnosis and therapy with micro- and nanometer-sized formulations. Methods, 2017; (130):4-13.

[141] Li H, Yang $\mathrm{Y}$, Zhang $\mathrm{M}$ et al. Acoustic Characterization and Enhanced Ultrasound Imaging of LongCirculating Lipid-Coated Microbubbles. J Ultrasound Med, 2017.

[142] Lindner J. Microbubbles in medical imaging: current applications and future directions. Nature Reviews Drug Discovery, 2004; (3):527-532.

[143] Hernot S, Klibanov AL. Microbubbles in ultrasoundtriggered drug and gene delivery. Adv Drug Deliv Rev, 2008; (60):1153-1166. 
[144] Wang S, Hossack JA, Klibanov AL. Targeting of microbubbles: contrast agents for ultrasound molecular imaging. J Drug Target, 2018; (26):420-434.

[145] DeJong N, Hoff L, Skotland T, Bom N. Absorption and scatter of encapsulated gas filled microspheres: theoretical considerations and some measurements. Ultrasonics, 1992; (30):95-103.

[146] Hoff L, Sontum PC, Hovem JM. Oscillations of polymeric microbubbles: Effect of the encapsulating shell. J Acoust Soc Am, 2000; (107):2272-2280.

[147] Qin S, Ferrara K. A model for the dynamics of ultrasound contrast agents in vivo Journal of the Acoustical Society of America, 2010; (128):1511-1521.

[148] Church C. The effects of an elastic solid surface layer on the radial pulsations of gas bubbles. Journal of the Acoustical Society of America, 1995; (97):1510-1521.

[149] Guo X, Li Q, Zhang Z et al. Investigation on the inertial cavitation threshold and shell properties of commercialized ultrasound contrast agent microbubbles. J Acoust Soc Am, 2013; (134):1622-1631.

[150] Sheeran PS, Dayton PA. Improving the performance of phase-change perfluorocarbon droplets for medical ultrasonography: current progress, challenges, and prospects. Scientifica (Cairo), 2014; (2014):579684.

[151] Sheeran PS, Luois S, Dayton PA, Matsunaga TO. Formulation and acoustic studies of a new phase-shift agent for diagnostic and therapeutic ultrasound. Langmuir, 2011; (27):10412-10420.

[152] Sheeran PS, Luois SH, Mullin LB et al. Design of ultrasonically-activatable nanoparticles using low boiling point perfluorocarbons. Biomaterials, 2012; (33):3262-3269.

[153] Sheeran PS, Matsunaga TO, Dayton PA. Phase change events of volatile liquid perfluorocarbon contrast agents produce unique acoustic signatures. Phys Med Biol, 2014; (59):379-401.

[154] Kripfgans O, Fowlkes J, Woydt $\mathrm{M}$ et al. In Vivo Droplet Vaporization for Occlusion Therapy and Phase Aberration Correction. IEEE Transactions on Ultrasonics Ferroelectrics \& Frequency Control, 2002; (49):726-738.

[155] Zhang M, Fabiilli ML, Haworth KJ et al. Initial investigation of acoustic droplet vaporization for occlusion in canine kidney. Ultrasound Med Biol, 2010; (36):16911703.

[156] Haworth K, Fowlkes J, Carson P, Kripfgans O. Towards aberration correction of transcranial ultrasound using acoustic droplet vaporization. Ultrasound Med Biol, 2008; (34):435-445.

[157] Huang J, Xu JS, Xu RX. Heat-sensitive microbubbles for intraoperative assessment of cancer ablation margins. Biomaterials, 2010; (31):1278-1286.

[158] Kang ST, Lin YC, Yeh CK. Mechanical bioeffects of acoustic droplet vaporization in vessel-mimicking phantoms. Ultrason Sonochem, 2014; (21):1866-1874.

[159] Miyoshi N, Kundu SK, Tuziuti T et al. Combination of Sonodynamic and Photodynamic Therapy against Cancer Would Be Effective through Using a Regulated Size of Nanoparticles. Nanoscience and Nanoengineering, 2016; (4):1-11.

[160] Abd El-Kaream SA, Abd Elsamie GH, Abd-Alkareem AS. Sono-photodynamic modality for cancer treatment using bio-degradable bio-conjugated sonnelux nanocomposite in tumor-bearing mice: Activated cancer therapy using light and ultrasound. Biochem Biophys Res Commun, 2018; (503):1075-1086.

[161] Wang P, Li C, Wang X et al. Anti-metastatic and proapoptotic effects elicited by combination photodynamic therapy with sonodynamic therapy on breast cancer both in vitro and in vivo. Ultrason Sonochem, 2015; (23):116-127.

[162] Wang H, Wang P, Zhang $\mathrm{K}$ et al. Changes in cell migration due to the combined effects of sonodynamic therapy and photodynamic therapy on MDA-MB-231 cells. Laser Physics Letters, 2015; (12):035603.

[163] Li Q, Wang X, Wang P et al. Efficacy of chlorin e6mediated sono-photodynamic therapy on 4T1 cells. Cancer Biother Radiopharm, 2014; (29):42-52.

[164] Chen HJ, Zhou XB, Wang AL et al. Synthesis and biological characterization of novel rose bengal derivatives with improved amphiphilicity for sono-photodynamic therapy. Eur J Med Chem, 2018; (145):86-95.

[165] Nomikou N, Curtis K, McEwan C et al. A versatile, stimulus-responsive nanoparticle-based platform for use in both sonodynamic and photodynamic cancer therapy. Acta Biomater, 2017; (49):414-421. 